\title{
Psychological Network Analysis of General Self- Efficacy in High vs Low Resilient Functioning Healthy Adults
}

Katja Schueler (katja.schueler@kgu.de) ${ }^{1,2}$, Jessica Fritz (jf585@cam.ac.uk) ${ }^{3}$, Lena Dorfschmidt (Id548@cam.ac.uk) ${ }^{3}$, Anne-Laura van Harmelen (a.van.harmelen@fsw.leidenuniv.nl) ${ }^{4}$, Eike Stroemer (eike.stroemer@lir-mainz.de) ${ }^{5}$, and Michèle Wessa (wessa@uni-mainz.de) $)^{1,5}$

1Department of Clinical Psychology and Neuropsychology, Institute of Psychology, Johannes Gutenberg-University of Mainz, Germany

${ }^{2}$ Medical Informatics Group, University Hospital of Frankfurt, Germany ${ }^{3}$ Department of Psychiatry, University of Cambridge, United Kingdom ${ }^{4}$ Institute of Education and Child Studies, University of Leiden, Netherlands ${ }^{5}$ Leibniz Institute for Resilience Research Mainz, Germany

Correspondence concerning this article should be addressed to:

Michèle Wessa, wessa@uni-mainz.de, Johannes Gutenberg-University Mainz, Department of Clinical Psychology and Neuropsychology, Institute for Psychology, Wallstraße 3, 55122 Mainz, Germany.

This research was funded by the Collaborative Research Center CRC 1193 'Neurobiology of Resilience to Stress-Related Mental Dysfunction' (Project C07) and the German Research Foundation DFG. 


\begin{abstract}
Resilience to stress has gained increasing interest by researchers from the field of mental health and illness and some recent studies have investigated resilience from a network perspective. General self-efficacy constitutes an important resilience factor. High levels of self-efficacy have shown to promote resilience by serving as a stress buffer. However, little is known about the role of network connectivity of self-efficacy in the context of stress resilience. The present study aims at filling this gap by using psychological network analysis to study self-efficacy and resilience. Based on individual resilient functioning scores, we divided a sample of 875 mentally healthy adults into a high and low resilient functioning group. To compute these scores, we applied a novel approach based on Partial Least Squares Regression on self-reported stress and mental health measures. Separately for both groups, we then estimated regularized partial correlation networks of a ten-item self-efficacy questionnaire. We compared three different global connectivity measures - strength, expected influence, and shortest path length - as well as absolute levels of self-efficacy between the groups. Our results supported our hypothesis that stronger network connectivity of self-efficacy would be present in the highly resilient functioning group compared to the low resilient functioning group. In addition, the former showed higher absolute levels of general self-efficacy. Future research could consider using partial least squares regression to quantify resilient functioning to stress and to study the association between network connectivity and resilient functioning in other resilience factors.

Keywords: resilience, network analysis, self-efficacy, connectivity, partial least squares regression, stress
\end{abstract}




\section{Introduction}

Stress resilience describes the maintenance or quick recovery of mental health during or after adverse events (Kalisch, Müller, \& Tüscher, 2014). Resilience research is part of a shift in perspective from a focus on psychopathology to the strengthening and maintaining of mental health. This is of great importance for preventing and overcoming the consequences of stress - as leading mental health organisations have already pointed out (Newman, 2005; Organization, 2013; Ziglio, 2017). Progress in stress resilience research comes with a need for appropriate research methods (Kalisch et al., 2019; Scheffer et al., 2018). A promising method in this context could be psychological network analysis since this methodology has already deepen our understanding of psychopathology and mental disorders (Borsboom, Cramer, Schmittmann, Epskamp, \& Waldorp, 2011). When it comes to stress resilience, networks of varying constitutions have been studied - for instance as statics resilience factors or dynamic hybrid symptom-and-resilience-factor networks (Borsboom et al., 2011; Cramer et al., 2016;

Fritz, Fried, Goodyer, Wilkinson, \& van Harmelen, 2018; Kalisch et al., 2019; Lunansky, van Borkulo, \& Borsboom, 2019). The present study builds on resilience factor networks and investigates the network structure of one resilience factor which is general selfefficacy. This means, we do not include psychopathology symptoms or other external factors in our networks. Instead, we relate the network structure of general self-efficacy to the level of resilient functioning to stress.

\section{Self-Efficacy as Resilience Factor}

Resilience factors are considered as promoters of stress resilience. They might come as external resources such as family or friendship support (Werner and Smith, 
1992). Further, they might emerge on an intra-individual level, for instance as selfesteem, emotion regulation skills (Fritz, de Graaff, Caisley, van Harmelen, and Wilkinson, 2018), self-compassion (Kaurin, Schönfelder and Wessa, 2018) and selfefficacy (Schwarzer \& Warner, 2013).

The resilience factor self-efficacy is an established psychological concept. It describes how strongly we believe in our ability to 'execute actions required to deal with prospective situations' (Bandura, 1977, 1982). Self-efficacy can be of general or context-specific manner, the latter e.g. with respect to coping abilities with severe diseases, parenting or caregiving, academic or leadership performance. Some researcher complementary discussed potential costs or detrimental effects of high selfefficacy particularly with respect to performance (Bandura \& Locke, 2003; Schönfeld, Preusser, \& Margraf, 2017). In the context of mental health and stress resilience, high self-efficacy seems to have a buffering effect on adverse events. Early studies found a dampening effect of high self-efficacy on the biological stress response when coping with phobic stressors (Bandura, Taylor, Williams, Mefford, \& Barchas, 1985; Wiedenfeld et al., 1990) and that low self-efficacy contributed to childhood depression (Bandura, Pastorelli, Barbaranelli, \& Caprara, 1999). When facing traumatic events, perceived coping self-efficacy served as mediator of posttraumatic recovery (Benight \& Bandura, 2004). Further, meta-analytic evidence revealed that high self-efficacy, among other positive expectations, mitigated PTSD symptoms (Gallagher, Long, \& Phillips, 2019). Longitudinal studies found this effect after generally traumatising events as well as single devastating events, e.g. after the 2004 Southeast Asian Tsunami natural disaster (Bosmans \& Van der Velden, 2015; Nygaard, Hussain, Siqveland, \& Heir, 2016). The 
buffering effect of self-efficacy also applies to daily stressors and their detrimental impact on mental health. In this context, high self-efficacy contributed to mental health and mitigated an increase in depressive and anxiety symptoms (Schönfeld, Brailovskaia, Zhang, \& Margraf, 2019; Schönfeld et al., 2017). To conclude, it is well accepted that high self-efficacy functions as stress-buffer and can therefore be considered a resilience factor (Bonanno, Brewin, Kaniasty and La Greca, 2010; Kalisch, Müller and Tüscher, 2014).

However, most studies have used absolute levels of self-efficacy and very little is known about the network structure of self-efficacy (Santos Jr, Kossakowski, Schwartz, Beeber, \& Fried, 2018) although such analytical approach has brought forward pathogenetic research (Borsboom et al., 2011). The present study tries to fill this gap by conceptualising self-efficacy as resilience factor network and relating its network properties to the resilient functioning to stress.

\section{Connectivity in Psychological Networks}

It is a common application of psychological network analysis to model symptoms of mental disorders (Borsboom et al., 2011). Often, such networks include psychopathology symptoms as nodes and their interrelations as edges. Network connectivity plays an important role in psychopathology networks. A simulation study showed that stronger connectivity, i.e. higher interrelations between network nodes, indicated worse prognosis of major depressive disorder (MDD) (Cramer et al., 2016). The authors used a pictorial example to illustrate the role of connectivity: strongly related symptoms could be seen as closely related domino blocks. When one domino 
block falls over, other blocks would easily fall over as well. That is the activation of one MDD symptom increases the likelihood that other symptoms will also be activated.

Overall, this mechanism makes the network more likely to switch into a depressed state and more difficult to reverse into a healthy state. Empirical studies have supported these findings: van Borkulo et al. (2017) found stronger connectivity in symptom networks of depression associated with more severe symptoms and worse prognosis two years later. Schweren, Borkulo, Fried, and Goodyer (2018) performed a conceptual replication in depressed adolescents. Global network connectivity was stronger in poor treatment responders, however this difference was not statistically different. In summary, empirical and stimulation studies have highlighted the importance of connectivity in psychopathology symptom networks. Strong connectivity seems related to the severity of mental illness. How this association behaves in the context of stress resilience is still unclear.

\section{Aim of this Study}

This study examines self-efficacy networks in the context of stress resilience and mental health. We investigate the network connectivity of the resilience factor selfefficacy in two groups that differ in their level of resilient functioning. In our study, stress resilience is conceptualised as the degree of mental health taking into account the individual stress history and it is measured independently of self-efficacy. Our hypotheses are inspired by findings from psychopathology symptom networks, which suggest that stronger connectivity in symptom networks is related to more severe psychopathology. We therefore hypothesize that stronger connectivity in selfefficacy networks should be associated with higher resilient functioning to stress. For 
the low-resilience group we therefore expect lower connectivity in self-efficacy networks. To the best of our knowledge, this is the first study that investigates network connectivity of the resilience factor general self-efficacy in relation to stress resilience. In addition, we apply a novel way to quantify resilient functioning based on partial least squares regression (Abdi, 2010). 


\section{Methods}

\section{Sample}

Participants were 875 (73\% women) undergraduate students $(M=21.97, S D=3.86)$ taking part in a longitudinal study investigating the effects of a blended resilience training. In the present study, baseline self-report data from all participants (irrespective of later group membership) collected between October 2017 and May 2019 were included. Exclusion criteria for study participation were any psychopharmacological medication, alcohol abuse, severe traumatic events (severe car accident, being sent to war, being held hostage, life-threatening natural disaster, sexual violence, or serious robbery), self-harm or suicidality, and psychotherapy during the last five years.

\section{Measures}

We used self-report measures to assess exposure to minor and major potentially stressful events, mental health, general self-efficacy, and demographic information. All instruments were administered in German.

Checklists of Minor and Major Stressors. We used two checklists to assess individual exposure to minor and major stressors. Stressors refer to any event or circumstance that could be perceived as especially stressful.

The Daily Hassles Checklist (DH) is a self-report of daily or minor stressors (Chmitorz et al., 2020). It assesses frequency and severity of 58 different minor stressors during the last seven days by asking a) 'On how many days did the event occur?' (number of days from 0 to 7 ) and b) 'How stressful was the event for you?' on a scale from 1 ('not at all') 
to 5 ('very much'). Examples of minor stressors are 'problems due to lack of help from others', 'commuting', or 'nightmares'.

The Life Events Checklist (LE) assesses life-time exposure to 27 major stressors. Participants indicate whether or not the event happened and, if so, how stressful they experienced it on a scale from 1 ('not at all') to 5 ('very much'). Examples for major stressors are 'death of a relative', 'sexual abuse', or 'job loss'. A complete list of all minor and major stressors can be found in the supplements.

Mental Health Questionnaires. Two self-report questionnaires served as indicators for current mental health: a) the Wellbeing Index, WHO-5, (World Health Organisation, 1998) and b) the Brief Symptom Inventory of psychopathology symptoms, BSI. The 5item World Health Organization Well Being Index (WHO-5) is a brief and popular questionnaire for mental well-being that has been translated into more than 30 languages (Topp, Østergaard, Søndergaard, \& Bech, 2015; WHO, 1998). Ratings of statements such as 'I have felt cheerful and in good spirits' over the last two weeks are assessed on a scale from 0 ('At not time') to 5 ('All of the time'). All items avoid clinical symptom-related language and focus primarily on positively phrased questions. The German version of the WHO- 5 demonstrates very good reliability with Cronbach's $\alpha=.92$ (Brähler, Mühlan, Albani, \& Schmidt, 2007) The German version of the Brief Symptom Inventory, BSI (Franke et al., 2011, 2017), is a short version of the Symptom Checklist SCL-90 (Derogatis \& Cleary, 1977; Derogatis \& Savitz, 1999). The scale consists of 18 psychopathology symptom items. Symptoms such as 'loneliness' or 'thoughts about death and dying' were rated on a scale from 1 ('not at all') and 5 ('very much') with respect to how often they bothered the participant during the last seven days. Six items 
each were combined to three distinct subscales: anxiety, depressive, and somatic symptoms. All subscales achieve good reliability indicated by Cronbach's $\alpha=.82$ for somatization, $\alpha=.87$ for depression, and $\alpha=.84$ for anxiety (Franke et al., 2017). To facilitate interpretation, we inverted the scores of all three BSI subscales; that is, higher values indicated lower levels of each anxiety, depression, and somatic symptoms.

Self-Efficacy Questionnaire. The General Self-efficacy Scale (Jerusalem \&

Schwarzer, 1992; Luszczynska, Scholz, \& Schwarzer, 2005) served as a measurement of self-efficacy expectancy. This unidimensional 10-item scale has demonstrated good reliability, with Cronbach's $\alpha$ ranging from .76 to .90 . Participants indicate on a scale from 1 ('not true') to 4 ('very true') how strongly they agree with general statements such as 'I can always manage to solve difficult problems if I try hard enough'. Table 1 shows a list of all 10 items translated from German into English.

\section{Statistical Analysis}

Summarizing the statistical procedure, we first calculated resilient functioning scores. We used partial least squares regression (PLSR) to predict mental health from stress history. From this model we extracted resilience scores as regression residuals. Based on these values we divided our sample into a highly resilient and a low resilient group. For both groups we then performed psychological network analysis. We calculated selfefficacy networks and compared the global connectivity between the groups. Additionally, we compared absolute levels of self-efficacy between both groups.

Data Pre-Processing. We prepared raw data for further analyses as follows. Four dependent variables were prepared for PLSR. We calculated a WHO-5 sum score and aggregated BSI items to three BSI subscale scores. The latter were inverted to facilitate 
interpretation in line with WHO-5 scores. This resulted in four continuous indicators of current mental health with higher values indicating high subjective well-being, low anxiety symptoms, low depressive symptoms, low somatic symptoms. Next, we zstandardised and normalised these scores and regressed out the effect of age and gender. Second, 147 independent variables were prepared for PLS regression. DH and LE items both included values below 0 on item level meaning that the corresponding event either did not happen at all or the participant did not wish to reply. All these values were set to exactly 0 .

Stress Resilience Scores. There is no established gold standard for quantifying stress resilience (Kalisch et al., 2014; Scheffer et al., 2018). One promising approach is to measure resilient functioning as doing better than expected given one's history of stress exposure (loannidis, Askelund, Kievit, \& van Harmelen, 2020; van Harmelen et al., 2017). van Harmelen et al. (2017) quantified resilience in a two-step approach, where first Principal Components Analysis is used to collapse stress exposure and mental health into one dimension respectively. Subsequently, the residuals of the regression of the summary mental health score on the summary stressor scores indicate the resilient functioning of mental health to stress. We combined these formerly two independent steps into one comprehensive model using a powerful statistical method, partial least squares regression (PLSR) (Abdi, 2010; Whitaker et al., 2016). To calculate stress resilient functioning scores a one component PLSR model has been applied. DH and LE were predictors and four mental health indicators (WHO-5, inverted BSI subscales) were outcome variables. The amount of variance in outcomes explained by the model was statistically significant compared to 1,000 permuted random models, i.e. it explains 
more variance in outcomes than expected by chance $(p<0.01)$. For each subject, we extracted the mean PLSR prediction residuals as resilient functioning scores to stress. Sample Split. Next, we split our sample into two groups and used 0 as the criterion for allocating the participants. The first group, the highly resilient group, consisted exclusively of participants who had a resilience score above 0 . The second group, the low resilient group, included only participants with a resilience score less than or equal to 0 .

Network Analysis. In both groups we calculated cross-sectional partial correlation networks of self-efficacy. Nodes of each network were the ten GSE questionnaire items. Network edges were LASSO-regularized partial correlations. These were computed as polychoric correlations which are suitable for ordinal data. The LASSO algorithm was used to regularise edges. This results in a rather sparse network by setting edges close to zero to exactly zero and hence exclude them from the network. We computed the following global network measures: a) average strength of all nodes - the strength of a single node is defined as the sum of all edges (absolute values) of this node; $b$ ) average expected influence of all nodes - the expected influence of a single node is defined as the sum of all edges taking into account the sign of an edge (relative values); c) average shortest path length - defined as the mean number of steps along the shortest paths for all possible pairs of network nodes (Costantini et al., 2015; Fritz, Fried, et al., 2018). In addition to that, we used the network comparison test (NCT), a permutation-based method that allows significance testing of global network strength (van Borkulo, 2018; van Borkulo et al., 2017). Even though NCT is the state-of-the-art of network 
comparison tests, it has not yet been validated for ordinal data. This limits the interpretation of NCT test results in our study.

Software. We used the programming language R 3.5 (R Core Team, 2019), the IDE RStudio 1.14, and Matlab 2018b (The MathWorks Inc.) for statistical analyses. Matlab PLS regression code was adapted from publicly available work by Whitaker et al. (2016). R code for network analysis strongly builds on the R packages 'bootnet' and 'qgraph'. Corresponding tutorials are provided by Epskamp, Borsboom, and Fried (2018) and Epskamp and Fried (2019). Resources for the 'NCT' R package is provided by van Borkulo (2018) and van Borkulo et al. (2017). All analysis scripts necessary to replicate the analysis of the present study will be available online upon peer-reviewed publication (https://osf.io/pz5ky). Raw and processed data are available upon request. 


\section{Results}

\section{Differences in Resilient Functioning to Stress}

As a sanity check, we tested expected differences in resilient functioning between both groups. A t-test confirmed that the two groups significantly differed in their resilience scores with a large effect size $(t=37.65, p<.000$, Cohen's $d=2.58)$. The highly resilient group ( $n=468,74 \%$ women, $M=1.81, S D=1.41$ ) scored significantly higher in stress resilience than the low resilient group ( $n=407,72 \%$ women, $M=-2.09, S D=1.62)$. See Table 3 for details. This confirms that the group split procedure had worked as expected.

\section{Differences in Absolute Levels of Self-Efficacy}

We compared absolute levels of self-efficacy between the groups using a t-test and Wilcoxon rank sum tests. The GSE total score was higher in the highly resilient group $(M=30.0, S D=3.9)$ than in the low resilient group $(M=27.2, S D=3.9)$. This difference was statistically significant with a medium effect size $(t=-10.3, p<0.000$, Cohen's $d=-0.7)$. To test for group differences in GSE items we used 10 Wilcoxon rank sum tests (two-sided, bonferroni corrected). Across all GSE items, highly resilient adults had significantly higher levels of self-efficacy than low resilient adults (all corrected $p<0.000$ ). See Figure 1 for general self-efficacy differences.

\section{Connectivity of Self-Efficacy Networks}

We expected three global network measures to differ between high and low resilient participants: average node strength, average node expected influence, and average shortest path length. As expected, strength and expected influence were higher in the high resilience group and shortest path length was lower in the high resilience group 
compared to the low resilience group (see Table 2). NCT confirmed differences in global strength on trend level $(P=0.06)$ with the limitation that this test has not been validated for ordinal data, only for binary and Gaussian data. Figure 2 shows the selfefficacy network of both groups. Network parameters remained qualitatively similar when based on unthresholded LASSO-regularization and on unregularized partial correlations (see Figures 3 and 4). 


\section{Discussion}

This study examined the global connectivity of a specific resilience factor network, i.e., self-efficacy. We compared self-efficacy networks between two groups: one that is high in stress resilience and with low stress resilience. As expected, our results confirm our hypothesis of a stronger network connectivity of self-efficacy in highly resilient compared to low resilient adults. At the global level, the former showed higher strength, higher expected influence, and shorter path length. Additionally, absolute values of selfefficacy, total score and single items, were higher in the highly resilient group. These results highlight the role of self-efficacy as resilience factor and demonstrate the importance of high connectivity between attributes of self-efficacy. To the best of our knowledge, this is the first study that takes stress resilience into account when investigating resilience factor networks.

\section{Contributions}

Our results contribute to resilience research in several ways. We provide evidence that network connectivity between attributes of a resilience factor, here general selfefficacy, and general stress resilience are inter-related (note: resilience factor networks and resilience scores were independently computed). Network connectivity has been shown to play an important role in psychopathology symptom networks (Cramer et al., 2016; Schweren et al., 2018; van Borkulo et al., 2017). It has been discussed as potential prognostic indicator for treatment response in major depression (Schweren et al., 2018; van Borkulo et al., 2017). Because the present study is based on cross-sectional data, we cannot make any statement regarding the prognostic value of connectivity in resilience factor networks. Still, in our 
study, the self-efficacy network had stronger connectivity in people with high stress resilience. In practical terms and in analogy to previous results of depression research (Borsboom et al., 2011), this could mean that in highly resilient people different attributes of self-efficacy are more interconnected, which could potentially enhance the combined effectiveness of the different facets of this resilience factor.

Besides network connectivity results, our findings underpin the importance of selfefficacy as resilience factor. This is in line with previous research showing that selfefficacy serves as buffer against potentially traumatic and daily stressors (Gallagher et al., 2019; Schönfeld et al., 2019). Moreover, self-efficacy has been identified as a moderator in the vulnerability-stress-model of anxiety; more precisely high self-efficacy buffers the impact of the gene (e.g., functional NPSR1 or 5-HTTLPR variation) $x$ environment (i.e., early adversity) interaction on anxiety symptoms (Schiele, Ziegler, Holitschke et al., 2016; Schiele, Herzog, Kollert et al., 2020).

Finally, the present study contributes to research methods for the investigation of stress resilience and resilience factors. Our approach combines a measure of stress resilience with the numerous opportunities of psychological network analysis (Epskamp et al., 2018; Epskamp \& Fried, 2019). The quantification of stress resilience as residual scores is particularly noteworthy. Building on existing research (van Harmelen et al., 2017), we provide further evidence that a residual score can be a promising option for calculating resilience in cross-sectional data.

\section{Limitations}

Some imitations should be considered when interpreting the present results. First, our results are limited to global connectivity. This means that they refer to self-efficacy as a 
whole and do not allow for the interpretation of individual self-efficacy facets. Our goal was to embed the concept of self-efficacy in the larger context of resilience. In our opinion, a single questionnaire item should therefore be given rather little weight in the broader context of resilience and resilience factors. Given the novelty of our approach, it however seemed reasonable to limit ourselves to global connectivity. Future studies might consider extending our approach to node-wise connectivity or other resilience factors.

Quantifying resilience as regression residuals comes with several mathematical challenges. Most important, resilience as regression residuals also include the error of the regression model. This could interfere with a clear interpretation of these scores as stress resilience. Future studies should consider these carefully before applying the 'residual approach'. Finally, we applied the Network Comparison Test, the state-ofthe-art method to test psychological networks for differences. However, the NCT has not been validated for ordinal data yet. We treated the ordinal self-efficacy items as Gaussian to run the NCT. This might have caused bias during the permutation process. Therefore, the NCT results have to be seen on an exploratory level. Further validation studies are needed as well as the development of network comparison tests suitable for ordinal data.

Finally, our study sample does not allow for generalization of results for several reasons. First, study participants were students of young age and therefore represent only a small part of the general population with respect to education and age. Second, gender distribution in our study was not equal with $70 \%$ female participants, which precluded analyses of gender effects. Future studies therefore have to replicate 
findings in a more representative and balanced sample with respect to gender distribution, age and education, in order to allow generalisability of results.

\section{Outlook}

Forthcoming studies could elaborate resilience factor networks and the role of their connectivity for stress resilience in greater depth. Stress resilience is a dynamic concept (Kalisch et al., 2017, 2014). Of particular interest would therefore be the prognostic value of network connectivity for the long-term course of stress resilience. In symptoms networks of major depression, the prognostic value of connectivity has already been shown (Schweren et al., 2018; van Borkulo et al., 2017). The corresponding question remains open whether connectivity of resilience factor networks can predict future stress resilience.

Future studies could investigate whether our results are transferable to other resilience factors such as emotion regulation skills or self-esteem. This might also involve the integration of different resilience factors into a network, similar to the study by Fritz, Fried, et al. (2018). In the case of hybrid networks (Kalisch et al., 2019), stress resilience scores could be helpful to integrate a measure of stress resilience as actual node in such a network. Finally, studies have shown that self-efficacy and other resilience factors can be increased in cognitive-behavioural interventions (Sahranavard, Esmaeili, Salehiniya, \& Behdani, 2019; Terp, Hjärthag, \& Bisholt, 2019). Future studies could consider the network perspective when evaluating the effectiveness of such interventions. 


\section{Conclusion}

To the best of our knowledge, this is the first to study the resilience factor self-efficacy using psychological network analysis. We were able to show that absolute levels as well as network connectivity of general self-efficacy is stronger in highly resilient than in low resilient healthy adults. Our results transfer findings from psychopathology networks to resilience research although a deeper understanding of our results has to be gained by future studies. In sum, our results undermine the importance of the relationship between connectivity in resilience factor networks and resilient functioning to stress. At the same time, we provide methodological inspiration for future research on resilience factor networks. 


\section{References}

Abdi, H. (2010). Partial least squares regression and projection on latent structure regression (PLS regression): PLS REGRESSION. Wiley Interdisciplinary Reviews: Computational Statistics, 2 (1), 97-106. doi: 10.1002/wics.51

Bandura, A. (1977). Self-efficacy: toward a unifying theory of behavioral change. Psychological Review, 84 (2), 191.

Bandura, A. (1982). Self-efficacy mechanism in human agency. American Psychologist, 37 (2), 122.

Bandura, A., \& Locke, E. A. (2003). Negative self-efficacy and goal effects revisited. Journal of Applied Psychology, 88 (1), 87.

Bandura, A., Pastorelli, C., Barbaranelli, C., \& Caprara, G. V. (1999). Self-efficacy pathways to childhood depression. Journal of Personality and Social Psychology, 76 (2), 258.

Bandura, A., Taylor, C. B., Williams, S. L., Mefford, I. N., \& Barchas, J. D. (1985). Catecholamine secretion as a function of perceived coping self-efficacy. Journal of Consulting and Clinical Psychology, 53 (3), 406.

Benight, C. C., \& Bandura, A. (2004). Social cognitive theory of posttraumatic recovery: The role of perceived self-efficacy. Behaviour Research and Therapy, 42 (10), 1129-1148.

Bonanno, G. A., Brewin, C. R., Kaniasty, K., \& La Greca, A. M. (2010). Weighing the Costs of Disaster: Consequences, Risks, and Resilience in Individuals, Families, and Communities. Psychological Science in the Public Interest, 11 (1), 1-49, https://doi.org/10.1177/1529100610387086 
Borsboom, D., Cramer, A. O. J., Schmittmann, V. D., Epskamp, S., \& Waldorp, L. J. (2011). The Small World of Psychopathology. PLOS ONE, 6 (11), e27407. doi: 10.1371/journal.pone.0027407

Bosmans, M. W., \& Van der Velden, P. G. (2015). Longitudinal interplay between posttraumatic stress symptoms and coping self-efficacy: A four-wave prospective study. Social Science \& Medicine, 134, 23-29.

Brähler, E., Mühlan, H., Albani, C., \& Schmidt, S. (2007). Teststatistische Prüfung und Normierung der deutschen Versionen des EUROHIS-QOL Lebensqualität-Index und des WHO-5 Wohlbefindens-Index. Diagnostica, 53 (2), 83-96.

Chmitorz, A., Kurth, K., Mey, L. K., Wenzel, M., Lieb, K., Tüscher, O., Kubiak, T. Kalisch, R. (2020). Assessment of microstressors in adults: Questionnaire development and ecological validation of the Mainz Inventory of Microstressors. JMIR Mental Health, 7 (2), e14566.

Costantini, G., Epskamp, S., Borsboom, D., Perugini, M., Mõttus, R., Waldorp, L. J., \& Cramer, A. O. J. (2015). State of the aRt personality research: A tutorial on network analysis of personality data in R. Journal of Research in Personality, 54, 13-29. doi: 10.1016/j.jrp.2014.07.003

Cramer, A. O. J., van Borkulo, C. D., Giltay, E. J., van der Maas, H. L. J., Kendler, K. S., Scheffer, M., \& Borsboom, D. (2016). Major Depression as a Complex Dynamic System. PLOS ONE, 11 (12), e0167490. doi: 10.1371/journal.pone. 0167490 
Derogatis, L. R., \& Cleary, P. A. (1977). Confirmation of the dimensional structure of the scl-90: A study in construct validation. Journal of Clinical Psychology, 33 (4), 981-989.

Derogatis, L. R., \& Savitz, K. L. (1999). The SCL-90-R, Brief Symptom Inventory, and Matching Clinical Rating Scales. In The use of psychological testing for treatment planning and outcomes assessment, 2nd ed (pp. 679-724).

Mahwah, NJ, US: Lawrence Erlbaum Associates Publishers.

Epskamp, S., Borsboom, D., \& Fried, E. I. (2018). Estimating psychological networks and their accuracy: A tutorial paper. Behavior Research Methods, 50 (1), 195-212. doi: 10.3758/s13428-017-0862-1.

Epskamp, S., \& Fried, E. I. (2019). bootnet: Bootstrap Methods for Various Network Estimation Routines.

Franke, G. H., Ankerhold, A., Haase, M., Jäger, S., Tögel, C., Ulrich, C., \& Frommer, J. (2011). Der Einsatz des Brief Symptom Inventory 18 (BSI-18) bei Psychotherapiepatienten. PPmP - Psychotherapie - Psychosomatik · Medizinische Psychologie, 61 (02), 82-86. doi: 10.1055/s-0030-1270518

Franke, G. H., Jaeger, S., Glaesmer, H., Barkmann, C., Petrowski, K., \& Braehler, E. (2017). Psychometric analysis of the brief symptom inventory 18 (BSI-18) in a representative German sample. BMC Medical Research Methodology, 17 (1), 14. doi: 10.1186/s12874-016-0283-3

Fritz, J., de Graaff, A. M., Caisley, H., van Harmelen, A.-L., \& Wilkinson, P. O. (2018). A Systematic Review of Amenable Resilience Factors That Moderate and/or Mediate 
the Relationship Between Childhood Adversity and Mental Health in Young People. Frontiers in Psychiatry, 9. doi: 10.3389/fpsyt.2018.00230

Fritz, J., Fried, E. I., Goodyer, I. M., Wilkinson, P. O., \& van Harmelen, A.-L. (2018). A Network Model of Resilience Factors for Adolescents with and without Exposure to Childhood Adversity. Scientific Reports, 8 (1), 15774. doi: 10.1038/s41598-018$34130-2$

Gallagher, M. W., Long, L. J., \& Phillips, C. A. (2019). Hope, optimism, self-efficacy, and posttraumatic stress disorder: A meta-analytic review of the protective effects of positive expectancies. Journal of Clinical Psychology, 76(3), 329-355. doi: 10.1002/jclp.22882.

loannidis, K., Askelund, A. D., Kievit, R. A., \& van Harmelen, A.-L. (2020). The complex neurobiology of resilient functioning after childhood maltreatment. BMC medicine, $18(1), 1-16$.

Jerusalem, M., \& Schwarzer, R. (1992). Self-efficacy as a resource factor in stress appraisal processes. In R. Schwarzer (Ed.), Self-efficacy: Thought control of action (pp. 195-213). Washington, DC, US: Hemisphere Publishing Corp.

Kalisch, R., Baker, D. G., Basten, U., Boks, M. P., Bonanno, G. A., Brummelman, E., Chmitorz, A., Fernàndez, G., Fiebach, C. J., Galatzer-Levy, I., Geuze, E., Groppa, S., Helmreich, I., Hendler, T., Hermans, E. J., Jovanovic, T., Kubiak, T., Lieb, K., Lutz, B., Müller, M. B., Murray, R. J., Nievergelt, C. M., Reif, A., Roelofs, K., Rutten, B. P. F., Sander, D., Schick, A., Tüscher, O., Diest, I. V., Harmelen, A. V., Veer, I. M., Vermetten, E., Vinkers, C. H., Wager, T. D., Walter, H., Wessa, M., Wibral, M. \& Kleim, B. (2017). The resilience framework as a strategy to combat 
stress-related disorders. Nature Human Behaviour, 1 (11), 784-790. doi: $10.1038 / \mathrm{s} 41562-017-0200-8$

Kalisch, R., Cramer, A. O. J., Binder, H., Fritz, J., Leertouwer, I., Lunansky, G., Meyer, B., Timmer, J., Veer, I. M. \& van Harmelen, A.-L. (2019). Deconstructing and reconstructing resilience: A dynamic network approach. Perspectives on Psychological Science, 14 (5), 765-777. doi: 10.1177/1745691619855637

Kalisch, R., Müller, M. B., \& Tüscher, O. (2014). A conceptual framework for the neurobiological study of resilience. Behavioral and Brain Sciences, 1-49. doi: $10.1017 / S 0140525 X 1400082 X$

Kaurin, A., Schönfelder, S., \& Wessa, M. (2018). Self-compassion buffers the link between self-criticism and depression in trauma-exposed firefighters. Journal of Counseling Psychology, 65(4), 453-462. https://doi.org/10.1037/cou0000275 Lunansky, G., van Borkulo, C. D., \& Borsboom, D. (2019). Personality, resilience, and psychopathology: A model for the interaction between slow and fast network processes in the context of mental health. Preprint.

Luszczynska, A., Scholz, U., \& Schwarzer, R. (2005). The General Self-Efficacy Scale: Multicultural Validation Studies. The Journal of Psychology, 139 (5), 439-457. doi: 10.3200/JRLP.139.5.439-457

Newman, R. (2005). Apa's resilience initiative. Professional psychology: Research and Practice, 36 (3), 227.

Nygaard, E., Hussain, A., Siqveland, J., \& Heir, T. (2016). General self-efficacy and posttraumatic stress after a natural disaster: a longitudinal study. BMC Psychology, 4 (1), 15. 
Word Health Organization (2013). WHO guidelines on conditions specifically related to stress. R Core Team. (2019). R: A Language and Environment for Statistical Computing. Vienna, Austria: R Foundation for Statistical Computing. Retrieved from https://www.R-project.org

Sahranavard, S., Esmaeili, A., Salehiniya, H., \& Behdani, S. (2019). The effectiveness of group training of cognitive behavioral therapy-based stress management on anxiety, hardiness and self-efficacy in female medical students. Journal of Education and Health Promotion, 8 .

Santos Jr, H. P., Kossakowski, J. J., Schwartz, T. A., Beeber, L., \& Fried, E. I. (2018). Longitudinal network structure of depression symptoms and self-efficacy in lowincome mothers. PloS One, $13(1)$, e0191675.

Scheffer, M., Bolhuis, J. E., Borsboom, D., Buchman, T. G., Gijzel, S. M. W., Goulson, D., Kammenga, J. E., Kemp, B., van de Leemput, I. A., Levin, S., Martin, C. M., Melis, R. J. F., van Nes, E. H., Romero, L. M. \& Olde Rikkert, M. G. M. (2018). Quantifying resilience of humans and other animals. Proceedings of the National Academy of Sciences of the United States of America, 115 (47), 11883-11890. doi: 10.1073/pnas.1810630115

Schiele, M. A., Herzog, K., Kollert, L., Schartner, C., Leehr, E. J., Böhnlein, J., Repple, J., Rosenkranz, K., Lonsdorf, T. B., Dannlowski, U., Zwanzger, P., Reif, A., Pauli P., Deckert, J. \& Domschke, K. (2020). Extending the vulnerability-stress model of mental disorders: three-dimensional NPSR $1 \times$ environment $\times$ coping interaction study in anxiety. British Journal of Psychiatry, 217(5), 645-650. doi: 10.1192/bjp.2020.73. 
Schiele, M. A., Ziegler, C., Holitschke, K., Schartner, C., Schmidt, B., Weber, H., Reif, A., Romanos, M., Pauli, P., Zwanzger, P., Deckert, J. \& Domschke, K. (2016). Influence of 5-HTT variation, childhood trauma and self-efficacy on anxiety traits: a gene-environment-coping interaction study. Journal of Neural Transmission, 123(8), 895-904. doi: 10.1007/s00702-016-1564-z.

Schönfeld, P., Brailovskaia, J., Zhang, X. C., \& Margraf, J. (2019). Self-efficacy as a mechanism linking daily stress to mental health in students: A three-wave crosslagged study. Psychological reports, 122 (6), 2074-2095.

Schönfeld, P., Preusser, F., \& Margraf, J. (2017). Costs and benefits of selfefficacy: differences of the stress response and clinical implications. Neuroscience \& Biobehavioral Reviews, 75, 40-52.

Schwarzer, R. \& Warner, L. M. (2013). Perceived self-efficacy and its relationship to resilience. In: S. Prince-Embury and D. H. Aklofske (Eds.), Resilience in Children, Adolescents and Adults: Translating Research into Practice (pp. 139-148). New York, US: Springer.

Schweren, L., Borkulo, C. D. v., Fried, E., \& Goodyer, I. M. (2018). Assessment of Symptom Network Density as a Prognostic Marker of Treatment Response in Adolescent Depression. JAMA Psychiatry, 75 (1), 98-100. doi: 10.1001/jamapsychiatry.2017.3561

Terp, U., Hjärthag, F., \& Bisholt, B. (2019). Effects of a cognitive behavioral-based stress management program on stress management competency, self-efficacy and self-esteem experienced by nursing students. Nurse Educator, 44 (1), E1-E5. 
Topp, C. W., Østergaard, S. D., Søndergaard, S., \& Bech, P. (2015). The WHO5 Well-Being Index: A Systematic Review of the Literature. Psychotherapy and Psychosomatics, 84 (3), 167-176. doi: 10.1159/000376585

van Borkulo, C. (2018). A tutorial on R package NetworkComparisonTest (NCT). In Symptom network models in depression research: From methodological exploration to clinical application (pp. 249-257).

van Borkulo, C., van Bork, R., Boschloo, L., Kossakowski, J., Tio, P., Schoevers, R., Borsboom, D. \& Waldorp, L. (2017). Comparing network structures on three aspects: A permutation test. 10.13140/RG.2.2.29455.38569.

van Harmelen, A.-L., Kievit, R. A., loannidis, K., Neufeld, S., Jones, P. B., Bullmore, E., R Dolan 3, NSPN Consortium, Fonagy, P. \& Goodyer, I. (2017). Adolescent friendships predict later resilient functioning across psychosocial domains in a healthy community cohort. Psychological Medicine, 47 (13), 2312-2322. doi: $10.1017 / S 0033291717000836$

Werner, E. E. \& Smith, R. S. (1992). Overcoming the Odds: High Risk Children from Birth to Adulthood. New York, Cornell University Press.

Whitaker, K. J., Vértes, P. E., Romero-Garcia, R., Váša, F., Moutoussis, M., Prabhu, G., Weiskopf, N., Callaghan, M. F., Wagstyl, K., Rittman, T., Tait, R., Ooi, C., Suckling, J., Inkster, B., Fonagy, P., Dolan, R. J., Jones, P. B., Goodyer, I. M., NSPN Consortium, \& Bullmore, E. T. (2016). Adolescence is associated with genomically patterned consolidation of the hubs of the human brain connectome. Proceedings of the National Academy of Sciences, 113 (32), 9105-9110. doi: 10.1073/pnas. 1601745113. 
WHO. (1998). Wellbeing measures in primary health care/the DEPCARE project (Tech. Rep. No. EUR/ICP/QCPH 0501 03). Stockholm, Sweden: World Health Organisation - Regional Office for Europe (https://www.euro.who.int/ data/assets/pdf file/0016/130750/E60246.pdf)

Wiedenfeld, S. A., O'Leary, A., Bandura, A., Brown, S., Levine, S., \& Raska, K. (1990). Impact of perceived self-efficacy in coping with stressors on components of the immune system. Journal of Personality and Social Psychology, 59 (5), 1082. 


\section{Supplements}

\section{List of minor stressors}

Here is a complete list of minor stressors that were included in the partial least squares model.

1. Loss or misplacement of items

2. Negative event in the media

3. Negative political event

4. Social obligation

5. Interruption in an activity

6. Waiting time or delay

7. Careless mistake or error due to lack of attention

8. Talk or blasphemy from other people

9. Discrimination or bullying by another person

10. Nightmares

11. Travel/ commuting to work/training place/ school/university

12. Minor offence (e.g. fine for misdemeanour)

13. Inconvenience with authority, office or other institution

14. Conflict or disagreement in the workplace

15. Conflict or disagreement with related parties

16. Conflict or disagreement between related parties

17. Conflict or disagreement with other unrelated persons

18. Conflict or disagreement with your child/children

19. Problem with childcare 
20. Procurement or transport service for others

21. Problem or inconvenience caused by your friends or relatives living too far away

22. Problem due to lack of support or help from others

23. Problem with your pet

24. Interference from unsafe environment

25. Impairment by dirt, contamination or stench

26. Problem due to not enough money available

27. Others owe you money

28. You owe money to others

29. High unexpected financial burden

30. Financial affairs

31. Unexpected or unwanted visit

32. Side effect of medication

33. Own physical complaint

34. Physical complaint

35. Lack of sleep or sleep problems

36. Doctor's visit

37. Office or clerical work at home

38. Housekeeping

39. Performing a minor repair

40. Problem with a technical device

41. Maintenance or servicing of an item

42. Unpleasant or bad weather 
43. Interfering behaviour or misconduct of others

44. Bad food

45. Noise

46. Traffic jam

47. Search for a parking space

48. Problem with a communication medium

49. Performance situation at work/school/study

50. High performance requirements or high workload in work/school/study

51. Boring activity

52. Meeting

53. Unregulated or excessive working hours

54. Problem with the planning or arrangement of appointments

55. Time pressure

56. Bad news

57. Problem or inconvenience caused by searching for a training/study/ or job

58. Problem or inconvenience due to house hunting or moving 


\section{List of majors stressors}

The following shows a full list of major stressors, i.e. significant life events that we used for the PLS model.

1. Job loss

2. Traumatic event at work

3. Marriage plans

4. House purchase, sale or removal

5. Major house renovation

6. Committing a theft or vandalism

7. Violations of the law

8. Serious financial problems

9. Serious illness, accident or diagnosis of illness by you or a close family member

10. Serious illness, accident or diagnosis of a close friend

11. Death of a family member

12. Death of a friend

13. Death of a beloved pet

14. Parental separation

15. Outstanding disputes between family members

16. Separation from boyfriend/girlfriend/partner/ spouse

17. Serious disputes with boyfriend/girlfriend/ partner/ spouse

18. Serious problems in friendships

19. Start of school of a child

20. Intensive care of an old or sick person 
21. Abortion at your or your partner's home

22. Serious physical illness - unable to work or perform daily activities

23. Injury - unable to work or engage in everyday activities

24. Pregnancy complications or miscarriage of you or your partner

25. Survival of physical abuse or robbery

26. Survival of sexual abuse

27. Any other adverse events (tests, car accident, house fire, earthquake, military action) 


\section{Tables and Figures}

Table 1: The General Self Efficacy Scale by Jerusalem \& Schwarzer (1992)

Item Statement

GSE 01 When resistance arises, I find ways and means to assert myself.

GSE 02 I always succeed in solving difficult problems if I make an effort.

GSE 03 I always succeed in solving difficult problems if I make an effort.

GSE 03 I have no difficulty in realizing my intentions and goals.

GSE 04 In unexpected situations I always know how to behave.

GSE 05 Even in unexpected events I believe that I can cope well with them.

GSE 06 I face difficulties calmly because I can always trust my abilities.

GSE 07 No matter what happens, I will always be able to cope.

GSE 08 For every problem I can find a solution.

GSE 09 If a new thing comes up to me, I know how to deal with it.

GSE 10 If a problem comes up, I can handle it on my own. 
Table 2

Global Connectivity of Self-Efficacy Networks

High Resilient Func Low Resilient Func

Strength

0.88

0.77

Expected influence

0.84

0.72

Shortest path length

7.43

9.07

Note. Comparison of global network connectivity measures in high resilient $(n=467)$ compared to low resilient $(n=408)$ adults. Values indicate the average connectivity parameters per node. 


\section{Table 3}

Characteristics of and Differences between Both Groups

\begin{tabular}{|c|c|c|c|}
\hline & High Resilience & Low Resilience & Difference \\
\hline Group size & $n=467$ & $n=408$ & \\
\hline Gender (female) & $n=344(74 \%)$ & $n=294(72 \%)$ & \\
\hline Age & $M=21.76(S D=3.42)$ & $M=22.20(S D=4.3)$ & n.s. \\
\hline$\Sigma$ minor stressors (freq) & $M=68.81(S D=32.76)$ & $M=65.12(S D=28.33)$ & n.s. \\
\hline$\Sigma$ minor stressors (sev) & $M=61.88(S D=30.58)$ & $M=60.46(S D=26.53)$ & n.s. \\
\hline$\Sigma$ major stressors & $M=19.84(S D=16.40)$ & $M=20.02(S D=15.49)$ & n.s. \\
\hline$\Sigma$ GSE & $M=59.88(S D=7.79)$ & $M=54.45(S D=7.93)$ & * \\
\hline GSE 01 & $M=3.25(S D=0.53)$ & $M=2.99(S D=0.52)$ & $\star C$ \\
\hline GSE 02 & $M=3.29(S D=0.56)$ & $M=3.10(S D=0.57)$ & ${ }^{*}{ }_{c}$ \\
\hline GSE 03 & $M=2.91(S D=0.67)$ & $M=2.59(S D=0.72)$ & $\star C$ \\
\hline GSE 04 & $M=2.55(S D=0.63)$ & $M=2.28(S D=0.74)$ & $* C$ \\
\hline GSE 05 & $M=2.96(S D=0.58)$ & $M=2.68(S D=0.65)$ & $\star C$ \\
\hline GSE 06 & $M=2.73(S D=0.75)$ & $M=2.32(S D=0.76)$ & ${ }^{*}{ }_{C}$ \\
\hline GSE 07 & $M=3.13(S D=0.70)$ & $M=3.01(S D=0.77)$ & ${ }^{*}{ }_{C}$ \\
\hline GSE 08 & $M=3.11(S D=0.68)$ & $M=2.85(S D=0.75)$ & ${ }^{*}{ }_{C}$ \\
\hline GSE 09 & $M=2.81(S D=0.59)$ & $M=2.56(S D=0.59)$ & $\star C$ \\
\hline GSE 10 & $M=3.02(S D=0.56)$ & $M=2.84(S D=0.56)$ & $\star C$ \\
\hline
\end{tabular}

Note. n.s. $=$ not significant with $p>0.05 ;{ }^{*}=p<0.000 ;{ }^{C}$ bonferroni corrected $p$-values 
Figure 1: Data distribution of all ten items of the General Self-Efficacy Scale (Jerusalem \& Schwarzer, 1992). Abbreviations GSE01 until GSE10 indicate item numbers. The $x$-axis shows the response ranging from 1 ('not at all') to 4 ('very much') and frequencies, i.e. number of participants, are depicted on the $y$-axis.

Group High Resilience Low Resilience
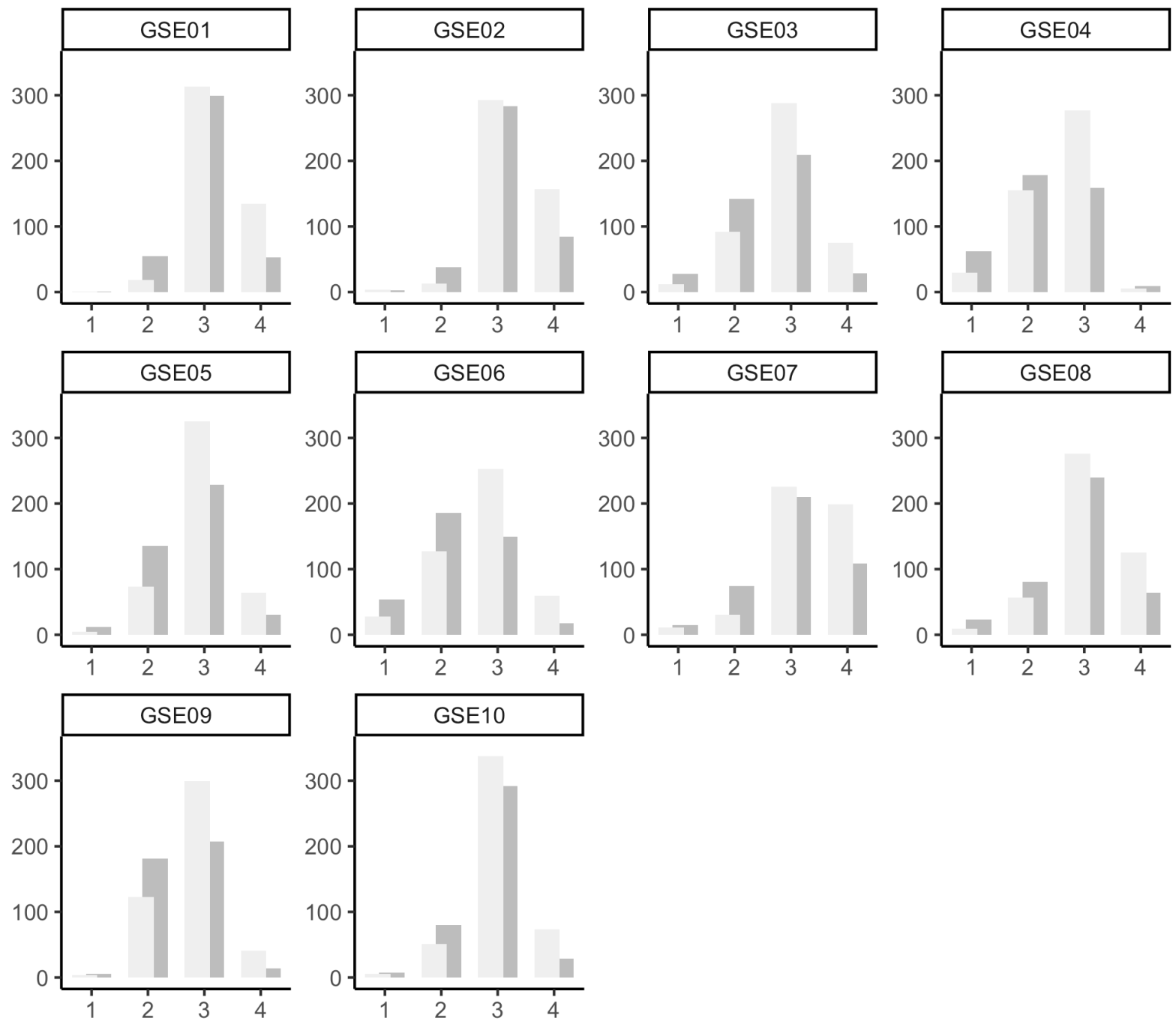
Figure 2: Regularized partial correlation networks of $A$ ) the high resilient functioning group ( $n=467)$ and $B)$ the low resilient functioning group $(n=408)$. Network nodes refer to the items of the General Self-Efficacy Scale (Jerusalem \& Schwarzer, 1992). Edges show regularized partial correlations between all ten questionnaire items (GSE01 until GSE10). A thicker line indicates higher correlations. No line indicates that the correlation between this respective pair of nodes was did not survive regularization.
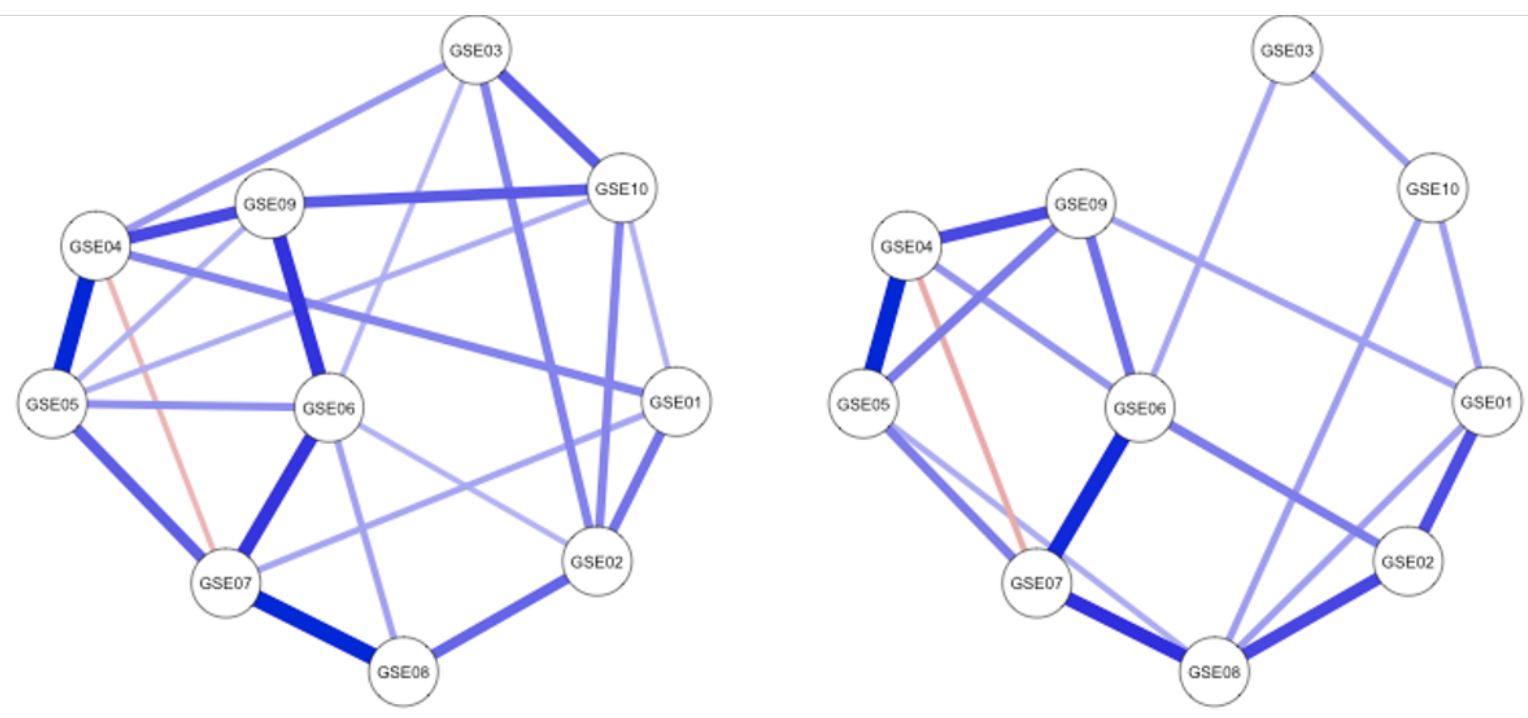
Figure 3: Supplementary NCT Results: These self-efficacy networks result from the network comparison test performed on bootnet partial correlation matrices ( $R$ package). Data were ordinally scaled. NCT has not been evaluated on ordinal data. A) Left network: high resilient functioning group, B) Right network: low resilient functioning group.

A) High Resilient Functioning Group

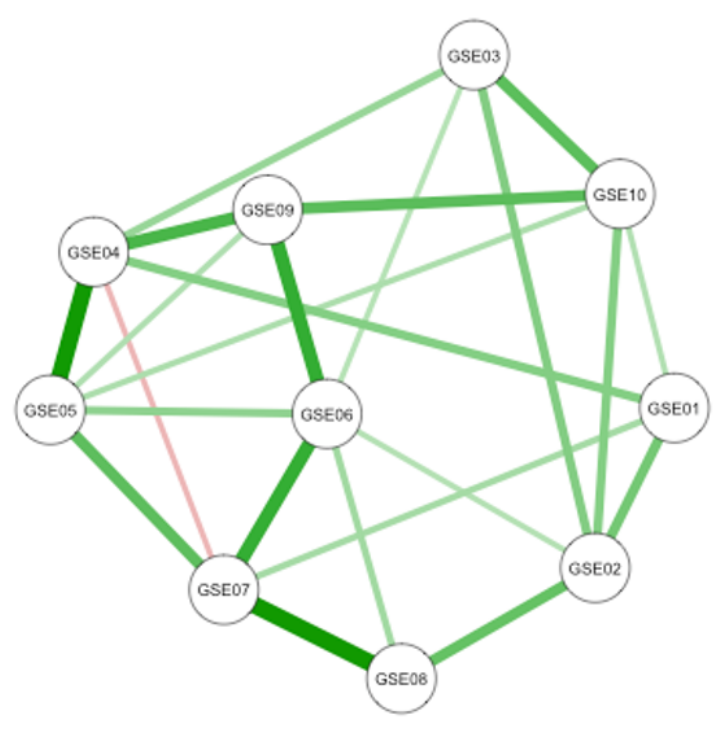

B) Low Resilient Functioning Group

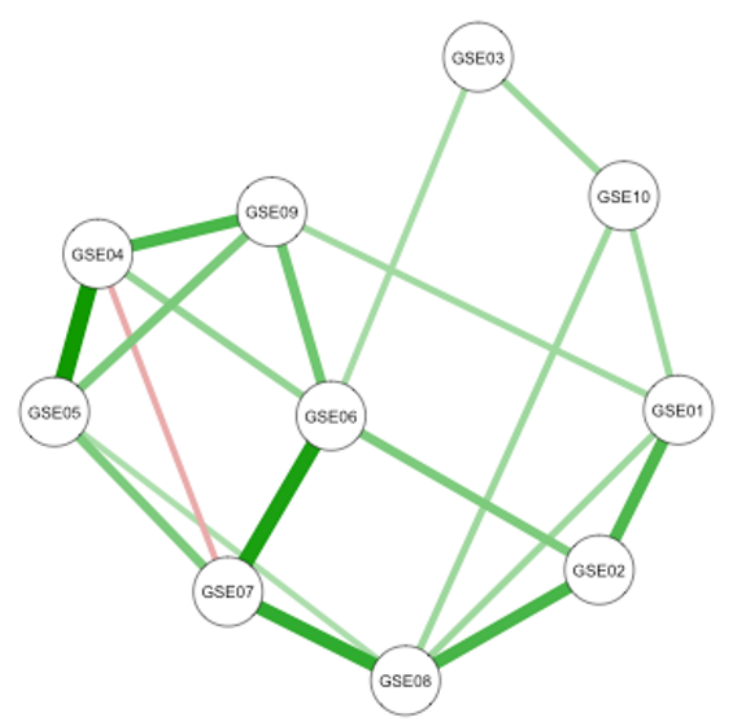


Figure 4: Supplementary NCT Results: These self-efficacy networks result from the network comparison test performed on raw data. Data were ordinally scaled. NCT has not been evaluated on ordinal data. A) Left network: high resilient functioning group, B) Right network: low resilient functioning group.

A) High Resilient Functioning Group

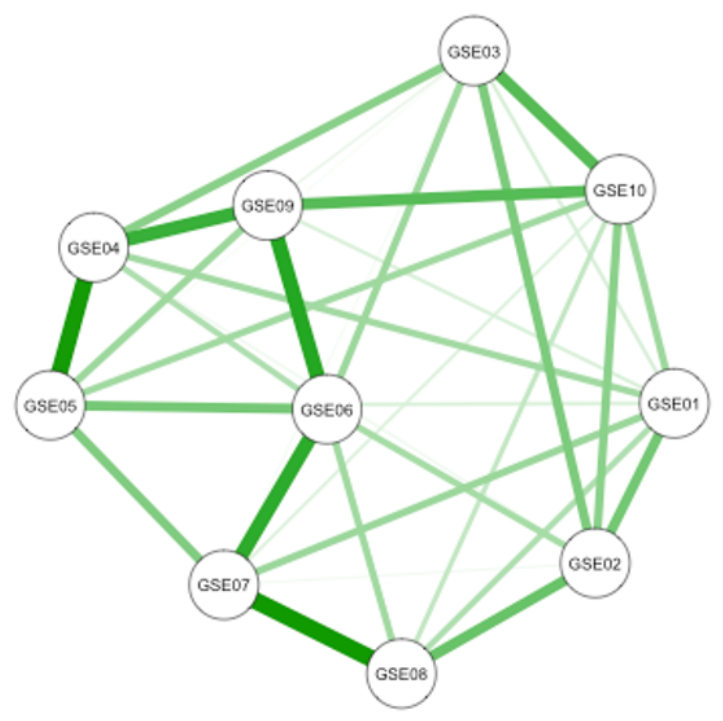

B) Low Resilient Functioning Group

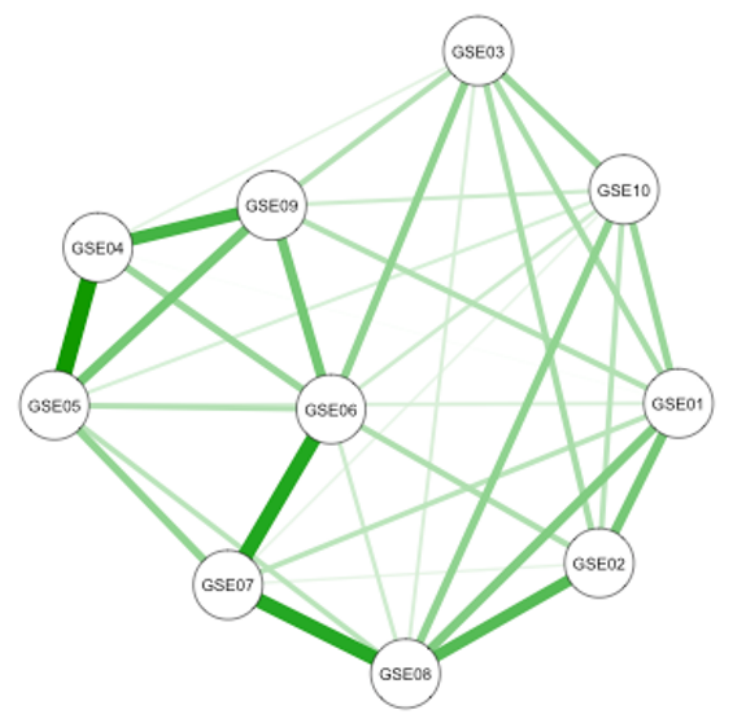

